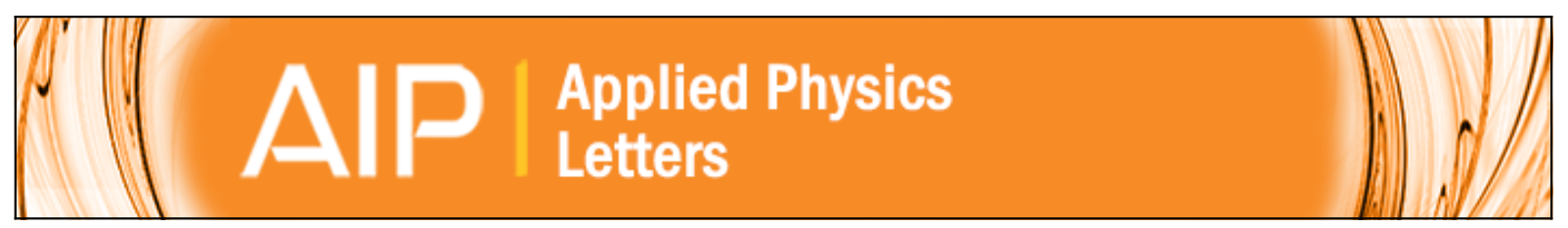

\title{
Direct mapping of plasmonic coupling between a triangular gold island pair
}

Weizhe Chen, Andrei Kirilyuk, Alexey Kimel, and Theo Rasing

Citation: Applied Physics Letters 100, 163111 (2012); doi: 10.1063/1.3700725

View online: http://dx.doi.org/10.1063/1.3700725

View Table of Contents: http://scitation.aip.org/content/aip/journal/apl/100/16?ver=pdfcov

Published by the AIP Publishing

\section{Articles you may be interested in}

Plasmonic emission enhancement of colloidal quantum dots in the presence of bimetallic nanoparticles

J. Appl. Phys. 115, 134315 (2014); 10.1063/1.4870575

Dynamic plasmonic tweezers enabled single-particle-film-system gap-mode Surface-enhanced Raman scattering Appl. Phys. Lett. 103, 191119 (2013); 10.1063/1.4829617

Optimization in interstitial plasmonic photothermal therapy for treatment planning

Med. Phys. 40, 103301 (2013); 10.1118/1.4810935

A microscopic study of strongly plasmonic $\mathrm{Au}$ and $\mathrm{Ag}$ island thin films

J. Appl. Phys. 113, 034302 (2013); 10.1063/1.4775784

Multimodal plasmon coupling in low symmetry gold nanoparticle pairs detected in surface-enhanced Raman scattering

Appl. Phys. Lett. 98, 183115 (2011); 10.1063/1.3555429

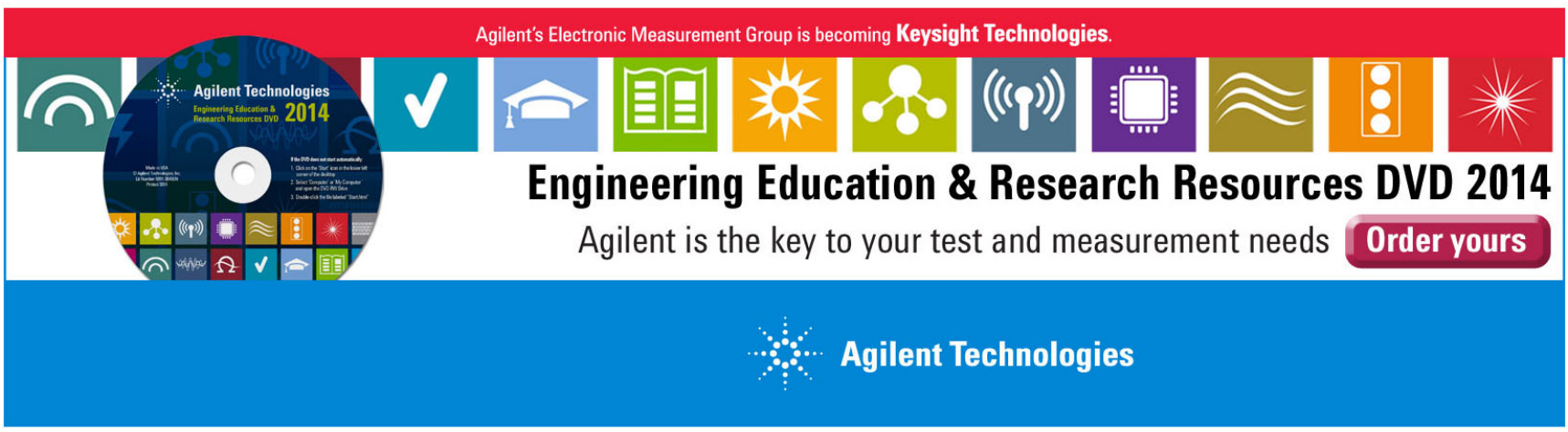




\title{
Direct mapping of plasmonic coupling between a triangular gold island pair
}

\author{
Weizhe Chen, ${ }^{\text {a) }}$ Andrei Kirilyuk, Alexey Kimel, and Theo Rasing \\ Institute for Molecules and Materials, Radboud University Nijmegen, Heyendaalseweg 135, \\ 6525 AJ Nijmegen, The Netherlands
}

(Received 7 January 2012; accepted 15 March 2012; published online 18 April 2012)

\begin{abstract}
Local plasmonic coupling between a triangular gold island pair is observed with high spatial resolution $(\sim 10 \mathrm{~nm})$ by an apertureless scanning near-field optical microscope. The measured local field distribution is fully reproduced by three dimensional numerical simulations. Our results show a strong near-field coupling between two adjacent gold triangles, which gives direct evidence to the local field enhancement in plasmonic nanoantenna effect. (C) 2012 American Institute of Physics. [http://dx.doi.org/10.1063/1.3700725]
\end{abstract}

Localized surface plasmon resonances (LSPRs) of metallic nanostructures have attracted much attention because of various promising applications, including optical nanoantennas, ${ }^{1,2}$ surface-enhanced Raman spectroscopy (SERS), ${ }^{3,4}$ enhanced linear and nonlinear photoluminescence processes, ${ }^{5,6}$ single molecular detection, ${ }^{7,8}$ universal plasmon ruler for precise gap distance measurement, ${ }^{9-11}$ and so on. The near-field interactions between two metallic nanoparticles are strongly dependent on the distance between the particle pair. ${ }^{2,12,13}$ These interactions will strongly enhance the local field at the gap between two adjacent particles, which could form different types of resonance optical antennas such as dipole antennas (rectangular shape $)^{1}$ and bowtie antennas (triangular shape). ${ }^{2}$

Since a strong interaction of their localized surface plasmon resonances of metallic nanoparticles only happens at very short distances (e.g., tens of nanometers), it is impossible to measure the enhanced local field with that sensitivity in the far field, due to the limitation of the spatial resolution of conventional far-field optical microscopes such as the two-photon photoluminescence (TPPL) measurement ${ }^{1,2}$ and total internal reflection (TIR) microscopy. ${ }^{14}$ Apertureless scanning near-field optical microscopy (apertureless SNOM), with the assistance of a sharp tip, has been applied to overcome these limitations and probe the plasmonic resonances of various metallic structures. ${ }^{15-17}$ However, direct near-field mapping at the gap between two strong interacting particles has not been achieved yet. In this paper, we report our direct mapping of plasmonic coupling between two triangular gold islands with our aperturelss SNOM. High spatial resolution down to $10 \mathrm{~nm}$ is obtained, showing clear plasmonic coupling at the narrow gap between two adjacent gold islands.

The apertureless SNOM experimental setup [schematically shown in Fig. 1(a)] is based on an atomic force microscope (AFM, Multimode, Veeco Inc.) operated at its tapping mode with full tip oscillation amplitude of $\sim 10 \mathrm{~nm}$. A silicon tip (Nanosensors, ATEC, $\sim 10 \mathrm{~nm}$ in radius) is used to avoid the strong influence of a metallic tip on the sample. ${ }^{18,19}$ Linearly polarized ( $p$-polarized with respect to the sample sur-

\footnotetext{
${ }^{\text {a) }}$ Author to whom correspondence should be addressed. Electronic mail: w.chen@science.ru.nl.
}

face) light at $800 \mathrm{~nm}$ from a Ti: Sapphire laser is focused onto the tip-sample junction at an angle of $30^{\circ}$ with respect to the sample surface via an aspheric lens. Both in-plane $\left(\left|E_{y}\right|\right)$ and out-of-plane $\left(\left|E_{z}\right|\right)$ components of the incident field interact with the sample. The focus size is $\sim 2 \mu \mathrm{m}$ in radius and the incident power is $\sim 4 \mathrm{~mW}$. Back-scattered light is collected by the same lens and detected by a heterodyne detection system ${ }^{20}$ with a frequency shifted $(\mathrm{M}=80 \mathrm{MHz})$ reference beam through an acousto-optical modulator (AOM). A second harmonic demodulation of the tip oscillation frequency $(\Omega \sim 250 \mathrm{kHz}$ ) is used to suppress the linear far-field background. ${ }^{15,21}$ Both near-field optical amplitude and phase information are extracted by demodulating the frequency of $M+2 \Omega$. The AFM topographic and optical images are simultaneously acquired while the sample is scanned in the $x-y$ plane as defined in Fig. 1(a).

The sample of triangular gold islands on a glass substrate is obtained by evaporating gold on a projection Fischer pattern in a hexagonal array with $230 \mathrm{~nm}$ periodicity. The typical size of the gold triangular islands is $\sim 100 \mathrm{~nm}$ and most of the islands are separated from each other with a distance of $\sim 100 \mathrm{~nm}$. Figure 1(b) shows the topography of a single gold island and its optical near-field distribution is shown in Fig. 1(c), exhibiting a local field enhancement at the top edge of this particle. The apertureless SNOM signal predominantly detects the out-of-plane $\left|E_{z-n f}\right|$ component of the local field because of the asymmetry of the AFM tip in the $z$ and $x / y$ directions. ${ }^{15,16}$ Thus, for a comparison, we use the CST microwave studio code based on a finite integration technique to simulate the optical near-field distribution of the gold islands, with a minimum mesh size of $1 \mathrm{~nm}$. The geometry and the excitation conditions are the same as that in the experiments with an angle of incidence of $60^{\circ}$ and $p$-polarization. The Drude model is applied for setting the permittivity of gold $(\epsilon \sim-25.8+1.6 i)$ at the excitation wavelength $(\lambda=800 \mathrm{~nm}){ }^{22}$ The calculated near-field $\left|E_{z-n f}\right|$ distribution of the single gold island is shown in Fig. 1(d), which is consistent with the experimentally obtained image.

Since the distances between most of the gold islands are relatively large $(>100 \mathrm{~nm})$, strong plasmonic interactions between particles cannot happen. However, some gold islands are very close to each other with a quite narrow gap below a few tens of nanometers, which provides the 

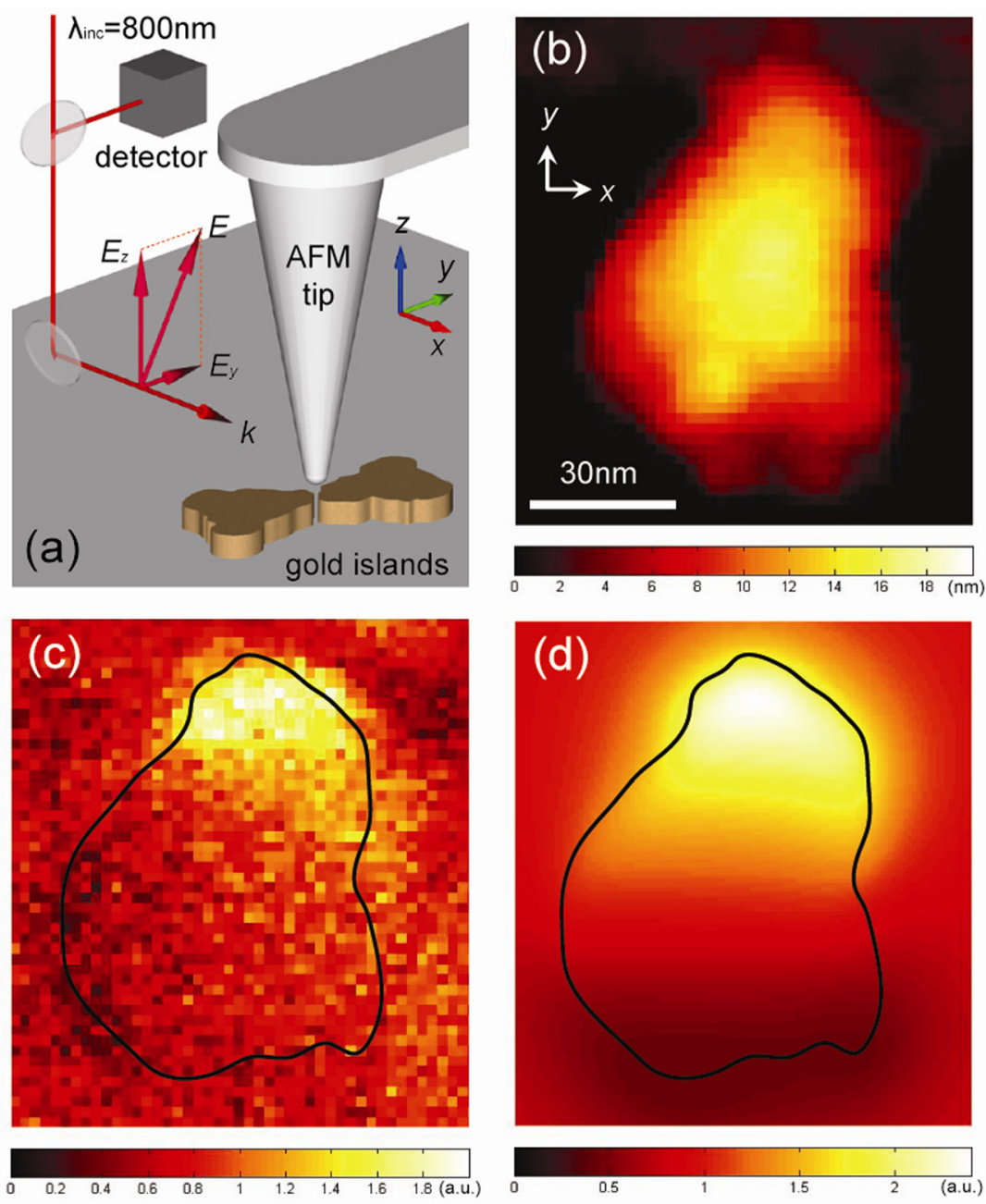

FIG. 1. (a) Schematic of the apertureless SNOM experiment for mapping the local field distribution of gold islands. Topography (a), measured optical amplitude (b), and simulated (c) z-component, $\left|E_{z-n f}\right|$, of the optical near-field images for a single triangular gold island. possibility for the particles to interact with each other. Figure 2(a) shows the topography of a typical triangular gold island pair with a gap of $\sim 10 \mathrm{~nm}$ between two particles. The simultaneously recorded optical amplitude image [Fig. 2(b)] exhibits the evanescent optical near-field distribution within tens of nanometers above the triangular gold island pair. At the gap of the triangular gold island pair, two bright areas are observed with a narrow dark slit $(\sim 10 \mathrm{~nm}$ in width) in between, which gives the evidence of the direct plasmonic coupling of these two islands, in their $E_{z-n f}$ component, at a very short distance. The coupling is further demonstrated by a phase jump of $\Phi \sim \pi$ from one particle to the other [Fig. 2(c)], that is, a sign change between the two islands due to the field coupling.

The observed plasmonic coupling between two adjacent gold islands can be simply understood as follows. When two metallic particles are far away from each other, their LSPR could be excited by proper incident wavelengths independently because LSPR are spatially confined within tens of nanometers around the particle surface, e.g., the single gold island in Fig. 1. When two particles are very close to each other, e.g., the triangular gold island pair in Fig. 2, the LSPR of one particle starts to feel that of the others. Due to the plasmonic coupling between the two adjacent particles, a new eigenmode of the joint LSPR is formed. Especially, when the size and shape of the particle pair is on resonance with the incident wavelength, a resonant optical nanoantenna is formed with a huge local field enhancement at the gap. ${ }^{1,2}$ Figure 2(d) schematically shows the interaction at the gap between two adjacent particles. In the space just above the gap, the local field is dominated by its in-plane component instead of the out-of-plane $z$-component, which explains the origin of the two bright areas with a narrow dark slit in between observed in the optical amplitude image at the gap [Fig. 2(b)]. The $\pi$ phase jump [Fig. 2(c)] is also from the local field coupling which changes the direction of $E_{z}$ from "up" at one particle to "down" at the others.

For a further comparison, the simulated $E_{z-n f}$-field distributions is shown in Fig. 3, reproducing both the experimental amplitude and phase distributions. It confirms the high-resolution images of the electric field distribution near the triangular gold island pair and shows the plasmonic coupling at the gap between the two islands. Fig. 4 shows the cross section profiles of the line $A \rightarrow B$ in Fig. 2, for both experiment ((a) and (b)) and simulation ((c) and (d)) results. They exhibit large spatial variations on a length scale as short as $10 \mathrm{~nm}$ for the optical amplitude and $6 \mathrm{~nm}$ for the optical phase, respectively.

In summary, our experiments reveal the direct plasmonic coupling of a gold triangular island pair with a narrow 

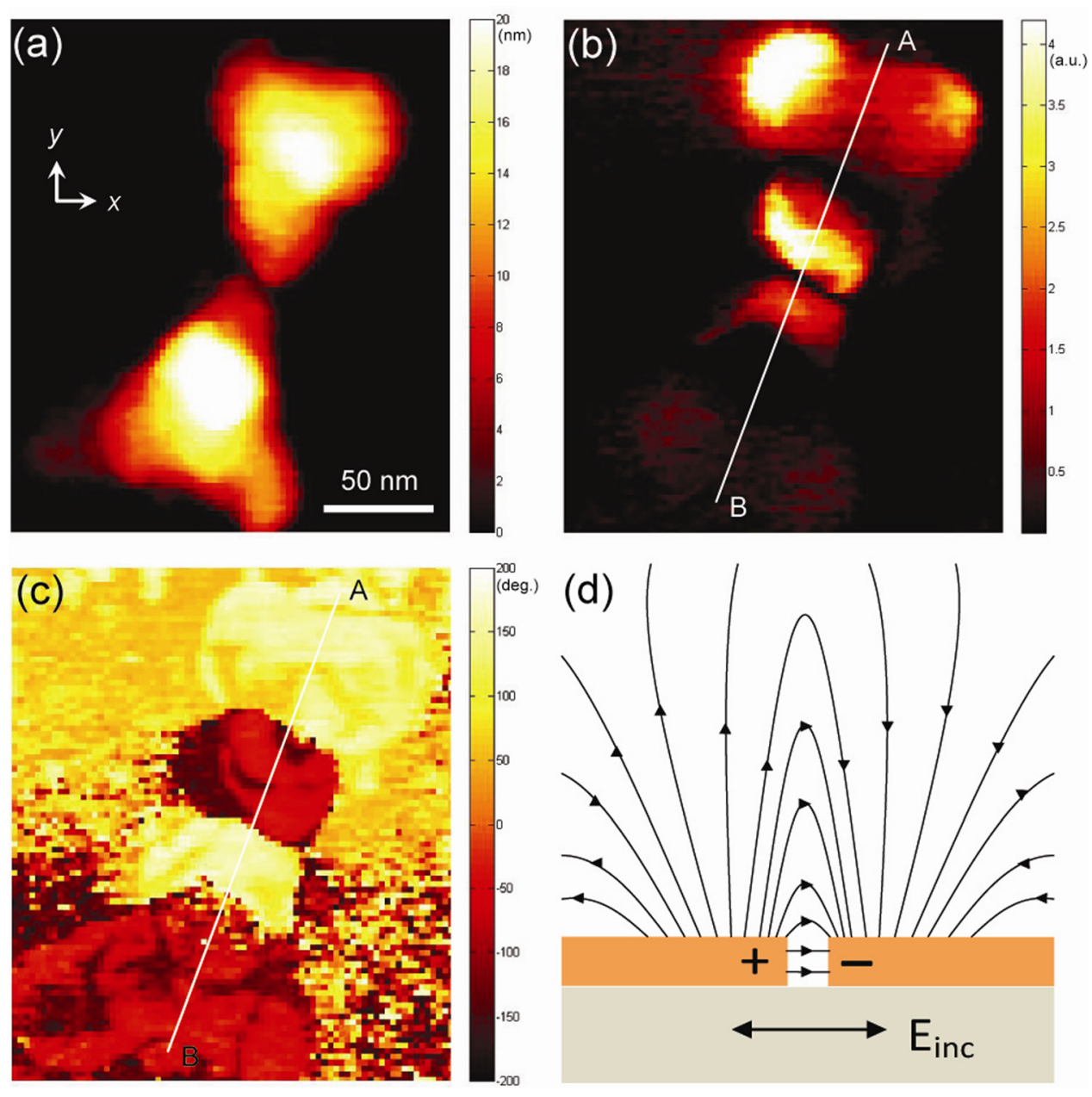

FIG. 2. Topography (a) and corresponding optical amplitude (b) and phase (c) images for a triangular gold island pair. The optical signal (b) and (c) is dominated by the out-of-plane $z$-component of the electric field, $\left|E_{z-n f}\right|$. Schematic (d) shows a field distribution model around a narrow gap between two particles. The incident polarization is parallel to the particle pair axis.

gap. The eigenmode of the gold triangular pair system is fully mapped with a spatial resolution as high as $\sim 10 \mathrm{~nm}$, which is in agreement with our three-dimensional numerical simulation. This observation provides detailed near-field distribution for understanding the interaction of adjacent nanoparticles, which is crucial for the optical nanoantenna effect. The high spatial sensitivity of the optical near-field distribu- tion of interacting nanoparticle systems emphasizes the importance of structure and shape control of plasmonic nanostructures for their nanophotonics applications, especially in optical nanoantennas. Finally, we expect further improvement with ultrasharp dielectric tips for a more precise full field mapping, especially for the strongly enhanced local field within the nanoantenna gaps.
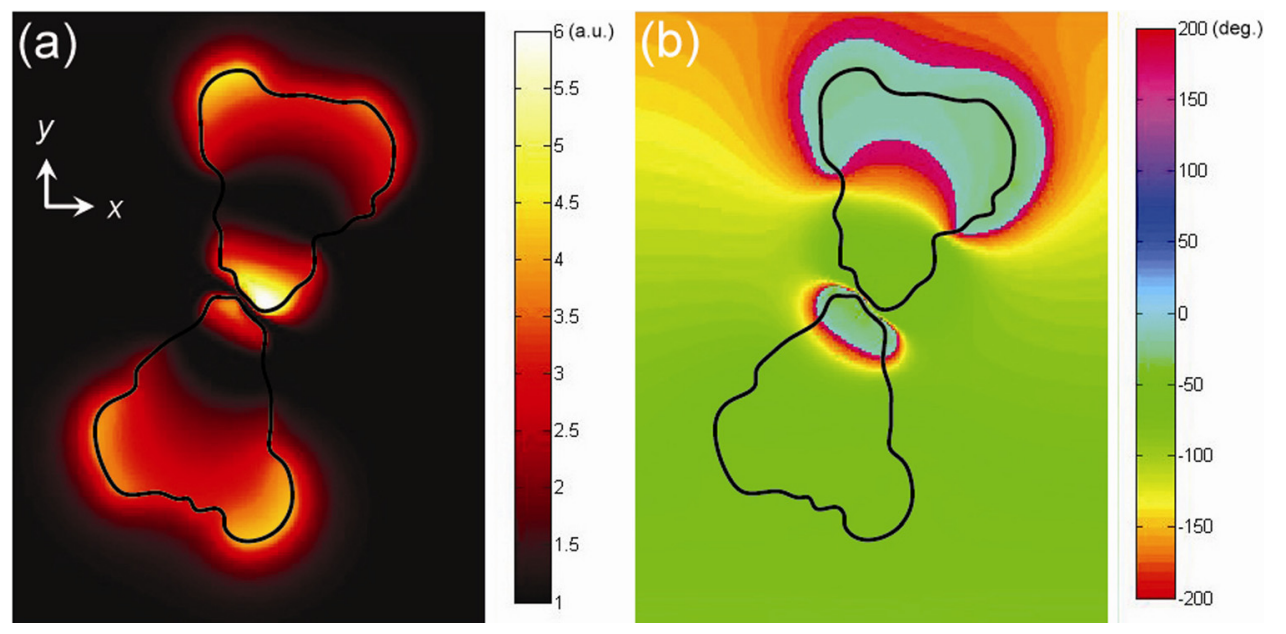

FIG. 3. Calculated z-component, $\left|E_{z-n f}\right|$, of the optical near-field at $10 \mathrm{~nm}$ above the triangular gold island pair for $800 \mathrm{~nm}$ excitation: (a) amplitude and (b) phase. The black contours show the shape of the structure with 20-nm thickness used for simulation. 

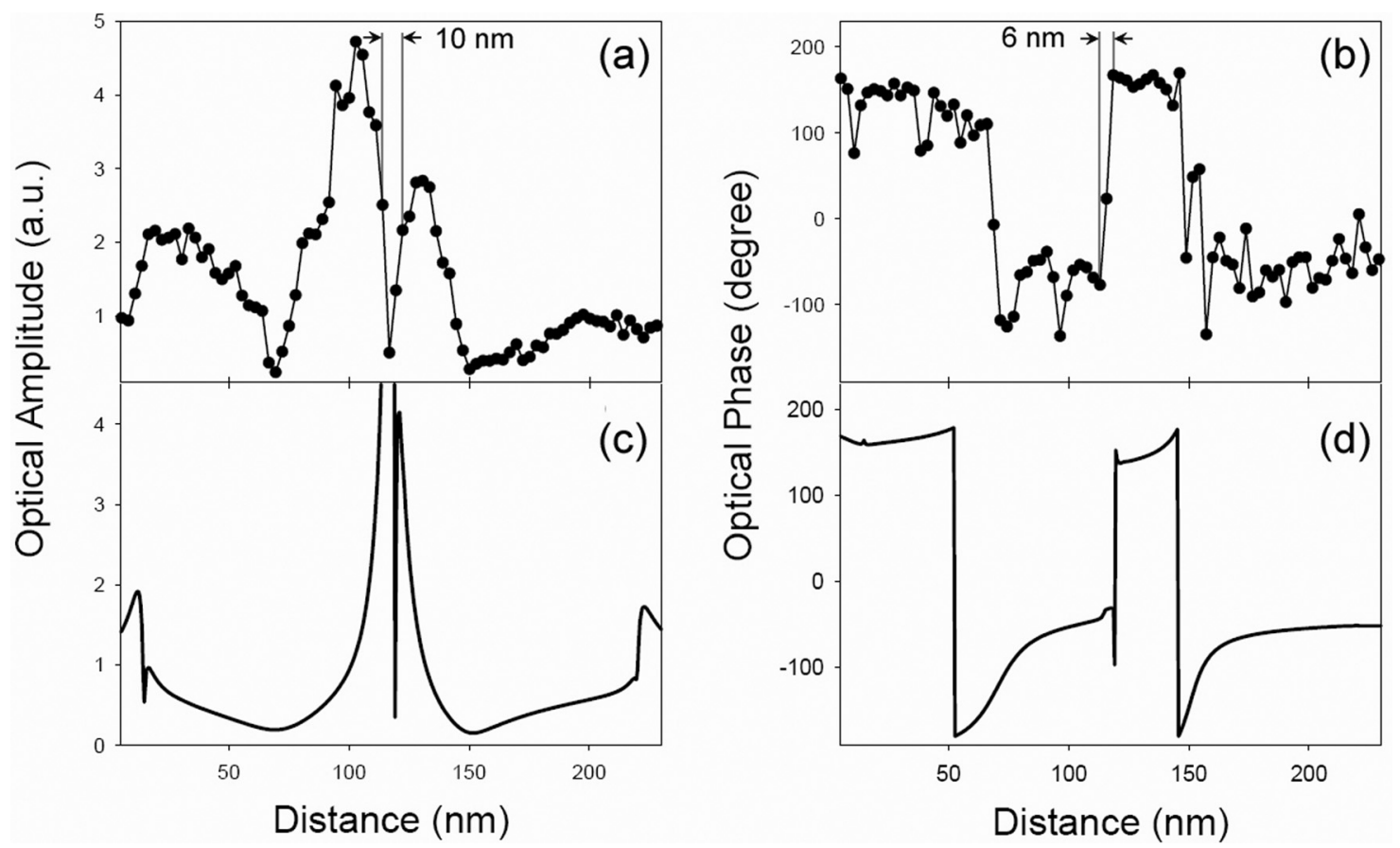

FIG. 4. The cross sections of the measured near-field optical amplitude (a) and phase (b) distributions along the cross lines $A \rightarrow B$ in Figs. 2(b) and 2(c), respectively, with a spatial field variation scale as short as $\sim 10 \mathrm{~nm}$ in experiment. Cross sections of the calculated field amplitude (c) and phase (d) along the same line as that in experiment.

This work was supported by NanoNed, The Netherlands Foundation for Fundamental Research on Matter (FOM), and Nederlandse Organisatie voor Wetenschappelijk Onderzoek (NWO). We thank A. F. van Etteger, A. J. Toonen, and J. W. Gerritsen for their technical support.

${ }^{1}$ P. Mühlschlegel, H. J. Eisler, O. J. F. Martin, B. Hecht, and D. W. Pohl, Science 308, 1607 (2005).

${ }^{2}$ P. J. Schuck, D. P. Fromm, A. Sundaramurthy, G. S. Kino, and W. E. Moerner, Phy. Rev. Lett. 94, 017402 (2005).

${ }^{3}$ C. Talley, J. Jackson, C. Oubre, N. Grady, C. Hollars, S. Lane, T. Huser, P. Nordlander, and N. Halas, Nano Lett. 5, 1569 (2005).

${ }^{4}$ Y. Cao, R. Jin, and C. Mirkin, Science 297, 1536 (2002).

${ }^{5}$ J. M. Gerton, L. A. Wade, G. L. Lessard, Z. Ma, and S. R. Quake, Phys. Rev. Lett. 93, 180801 (2004).

${ }^{6}$ N. Hayazawa, K. Furusawa, A. Taguchi, S. Kawata, and H. Abe, Appl. Phys. Lett. 94, 193112 (2009).

${ }^{7}$ A. M. Michaels, J. Jiang, and L. J. J. Brus, Phys. Chem. B 104, 11965 (2000).

${ }^{8}$ J. P. Camden, J. A. Dieringer, Y. Wang, D. J. Masiello, L. D. Marks, G. C. Schatz, and R. P. V. Duyne, J. Am. Chem. Soc. 130, 12616 (2008).
${ }^{9}$ B. M. Reinhard, M. Siu, H. Agarwal, A. P. Alivisatos, and J. Liphardt, Nano Lett. 5, 2246 (2005).

${ }^{10}$ P. K. Jain, W. Huang, and M. A. El-Sayed, Nano Lett. 7, 2080 (2007).

${ }^{11}$ C. Sönnichsen, B. M. Reinhard, J. Liphardt, and A. P. Alivisatos, Nat. Biotechnol. 23, 741 (2005).

${ }^{12}$ A. M. Funston, C. Novo, T. J. Davis, and P. Mulvaney, Nano Lett. 9, 1651 (2009).

${ }^{13}$ I. Romero, J. Aizpurua, G. W. Bryant, and F. J. García de Abajo, Opt. Express 14, 9988 (2006).

${ }^{14}$ S. C. Yang, H. Kobori, C. L. He, M. H. Lin, H. Y. Chen, C. Li, M. Kanehara, T. Teranishi, and S. Gwo, Nano Lett. 10, 632 (2010).

${ }^{15}$ M. Rang, A. C. Jones, F. Zhou, Z. Y. Li, B. J. Wiley, Y. Xia, and M. B. Raschke, Nano Lett. 8, 3357 (2008).

${ }^{16}$ R. Esteban, R. Vogelgesang, J. Dorfüller, A. Dmitriev, C. Rockstuhl, C. Etrich, and K. Kern, Nano Lett. 8, 3155 (2008).

${ }^{17}$ D. S. Kim, J. Heo, S. H. Ahn, S. W. Han, W. S. Yun, and Z. H. Kim, Nano Lett. 9, 3619 (2009).

${ }^{18}$ J. Sun, P. S. Carney, and J. Schotland, J. Appl. Phys. 120, 103103 (2007).

${ }^{19}$ A. Garcia-Etxarri, I. Romero, F. J. Garcia de Abajo, R. Hillenbrand, and J. Aizpurua, Phy. Rev. B 79, 125439 (2009).

${ }^{20}$ R. Hillenbrand and F. Keilmann, Phy. Rev. Lett. 85, 3029 (2000).

${ }^{21}$ F. Keilmann and R. Hillenbrand, Philos. Trans. R. Soc. London, Ser. A 362, 787 (2004).

${ }^{22}$ P. B. Johnson and R. W. Christy, Phys. Rev. B 6, 4370 (1972). 